\title{
The Variational Approach for P-Laplace Equation with Lack of Compactness
}

\author{
Dong Zhang* \\ Department of Mathematics, Cangzhou Normal College, Hebei 061000, China \\ *Corresponding author
}

\begin{abstract}
Minimization technique is used on the Nehari manifold for $p$-Laplace equation with a nonhomogeneous nonlinearity where compactness is not guaranteed. In the space of radial functions, the point overcoming the lack of compactness is to show that the minimizing sequence converges in a strong enough sense to pass to the limit in the nonlinear term. The nontrivial radial solution is found by restoring compactness in such space.
\end{abstract}

Keywords- p-Laplace equation; variational method; Nodal solution; Nehari manifold

MR(2010) Subject Classification : O175.29

\section{INTRODUCTION}

There is by now a vast literature on p-Laplacian. A good starting point is Garcia Azorero and Peral ${ }^{[1]}$. A systematic treatment of many questions concerning the p-Laplacian can be found in the book by Lindqvist ${ }^{[2]}$ and Fan ${ }^{[3]}$. Recently the existence and nonexistence by virtue of critical point theory are discussed in ${ }^{[4-6]}$. The method of minimization on the Nehari manifold ${ }^{[7-9]}$ is particularly useful for nonhomogeneous nonlinearities, for which minimization on spheres does not apply. A rather detailed exposition can be found in the books by Willem ${ }^{[10]}$, and Kuzin - Pohožaev ${ }^{[11]}$; the application given in ${ }^{[1]}$ concerns problems on unbounded domains. The reasons for this often come from geometrical or physical aspects of the problem, in ${ }^{[12]}$, Struwe introduced application to nonlinear partial differential equation an Hamiltonian systems via variational method. Badiale and Serra ${ }^{[13]}$ present some examples where compactness is not guaranteed a priori. The lack of compactness can take different forms, but in the simplest case, it is manifest through the fact that minimizing sequences are maybe bounded, but not (pre-)compact in the function spaces where the problem is set. Here we confine ourselves to some more or less simple example with lack of compactness which takes place when one works in unbounded open sets $R^{N}$. The reason for this is the invariance of $R^{N}$ with respect to translations, which in turn makes the embedding of $W^{1, p}$ where $1<p<N$ into $L^{q}\left(R^{N}\right)$ not compact for any $q$ where $\frac{N p}{N-p}>q>p$. A natural attempt overcome this problem is to guess that translational invariance is the only reason for the failure of compactness, and to try to work in a space of functions where translations are not allowed. This is possible in this case because the problem is also invariant under rotations, so that one can try to work in spaces of radial functions ${ }^{[14-15]}$.

\section{PRELIMINARIES}

We treat the p-Laplacian equation on $R^{N}$ with $N \geq 3$ :

$$
\left\{\begin{array}{l}
-\Delta_{p} u=|u|^{q-2} u+\lambda|u|^{r-2} u \quad \text { in } R^{N}, \\
u \in W^{1, p}\left(R^{N}\right)
\end{array}\right.
$$

where $\lambda \in R, 1<p<N$ and $p<r<q<p^{*}$. A weak solution of (1) is a function $u \in W^{1, p}$ such that $\forall v \in W^{1, p}\left(R^{N}\right)$

$$
\int_{R^{N}}|\nabla u|^{p-2} \nabla u \nabla v d x=\int_{R^{N}}|u|^{q-2} u v d x+\lambda \int_{R^{N}}|u|^{r-2} u v d x
$$

Define $W_{r}=\left\{u \in W^{1, p}\left(R^{N}\right) \mid u\right.$ is radial $\}$. More precisely, we give a pointwise estimate for functions in $W_{r}$.

Lemma 2.1 There exists a constant $C>0$ such that for every $u \in W_{r}$

$$
|u(x)| \leq C \frac{\|u\|}{|x|^{\frac{N-p}{p}}} \quad \text { for every } \quad x \neq 0
$$

We prove that the heuristic idea that translations are the only obstruction to compactness is correct. The Sobolev inequalities on $R^{N}$ show that $W_{r}$ is continuously embedded into $L^{q}\left(R^{N}\right)$ for $\frac{N p}{N-p} \geq q \geq p$. The following lemma proves this embedding is compact if $\frac{N p}{N-p}>q>p$.

Lemma $2.2^{[16]}$ (Rellich-Kondrachov Compactness Theorem) Assume $U$ is a bounded open subset of $R^{N}$ and $\partial U$ is $C^{1}$. Suppose $1<p<N$. Then $W^{1, p}(U)$ is compactly embedded in $L^{q}(U)$ for $1<q<p^{*}$.

Lemma 2.3 Let $q \in\left(p, p^{*}\right)$. Then the embedding of $W_{r}$ into $L^{q}\left(R^{N}\right)$ is compact. 
Proof It is enough to show that if $\left\{u_{k}\right\}_{k}$ is a sequence in $W_{r}$ such that $u_{k} \rightarrow 0$, then $u_{k} \rightarrow 0$ in $L^{q}\left(R^{N}\right)$. From Lemma 2.2, assume

$$
u_{k} \rightarrow 0 \text { in } L_{l o c}^{q}\left(R^{N}\right) \text { and } \quad u_{k} \rightarrow 0 \quad \text { a.e. }
$$

Let $B=\left\{x \in R^{N}|| x \mid<1\right\}$ be the unit ball of $R^{N}$.We can get

$$
\int_{R^{N}}\left|u_{k}\right|^{q} d x=\int_{B}\left|u_{k}\right|^{q} d x+\int_{B^{c}}\left|u_{k}\right|^{q} d x=\int_{B^{c}}\left|u_{k}\right|^{q} d x+o(1)
$$

Fix $l>p^{*}$,by Lemma 2.1, in $B^{c}$ we have, for some appropriate constant $C$,

$$
\left|u_{k}(x)\right|^{l} \leq \frac{C}{|x|^{\frac{N-p}{p}}}
$$

And $l \frac{N-p}{p}>N$. This shows that $\frac{1}{|x|^{\frac{l-p}{p}}} \in L^{1}\left(B^{c}\right)$, and then , by dominated convergence

$$
\int_{B^{c}}\left|u_{k}\right|^{l} d x \rightarrow 0
$$

Let now $t \in(0,1)$ be such that $q=p t+l(1-t)$; by the Hölder inequality we can write

$$
\int_{B^{c}}\left|u_{k}\right|^{q} d x=\int_{B^{c}}\left|u_{k}\right|^{p t}\left|u_{k}\right|^{l(1-t)} d x \leq\left(\int_{B^{c}}\left|u_{k}\right|^{p} d x\right)^{t}\left(\int_{B^{c}}\left|u_{k}\right|^{l} d x\right)^{(1-t)} .
$$

Since the sequence $\left\{\int_{B^{c}}\left|u_{k}\right|^{p} d x\right\}_{k}$ is bounded and $\int_{B^{c}}\left|u_{k}\right|^{l} d x$ $\rightarrow 0$, we immediately obtain $\int_{B^{c}}\left|u_{k}\right|^{q} d x \rightarrow 0$, so that

$$
\int_{R^{N}}\left|u_{k}\right|^{q} d x \rightarrow 0
$$

Lemma 2.4 ${ }^{[15]}$ For every $u \in W^{1, p}\left(R^{N}\right), u \geq 0$, there exists $u^{*} \in W_{r}, u^{*} \geq 0$, for all $p, q>1$

$$
\int_{R^{N}}\left|\nabla u^{*}\right|^{p} d x \leq \int_{R^{N}}|\nabla u|^{p} d x \text { and } \int_{R^{N}}\left|u^{*}\right|^{q} d x \leq \int_{R^{N}}|u|^{q} d x
$$

\section{Main Results}

Theorem 3.1 Problem (1) admits at least one nontrivial nonnegative solution.
This result will be obtained by a sequence of lemmas. We equip $W^{1, p}\left(R^{N}\right)$ with the norm

$$
\|u\|^{p}=\int_{R^{N}}|\nabla u|^{p} d x
$$

And we look for a solution as a minimize of the associated functional constrained on the Nehari manifold. So we define the functional $I: W^{1, p}\left(R^{N}\right) \rightarrow R$ as

$$
I(u)=\frac{1}{p} \int_{R^{N}}|\nabla u|^{p} d x-\frac{1}{q} \int_{R^{N}}|u|^{q} d x-\frac{\lambda}{r} \int_{R^{N}}|u|^{r} d x
$$

And the Nehari Manifold

$$
\begin{gathered}
\mathcal{N}=\left\{u \in W^{1, p} \mid u \neq 0, I^{\prime}(u) u=0\right\} \\
=\left\{\left.u \in W^{1, p}\left|u \neq 0,\|u\|^{p}=\right| u\right|_{q} ^{q}+\lambda|u|_{r}^{r}\right\}
\end{gathered}
$$

If $u \in \mathcal{N}$, then $I(u)>\left(\frac{1}{p}-\frac{1}{r}\right)\|u\|^{p}>0$.

Lemma 3.2 ${ }^{[13]}$ The Nehari manifold is not empty.

Define $m=\inf _{u \in \mathcal{N}} I(u)$ and try to show that $m$ is attained by some $u \in \mathcal{N}$.

Lemma 3.3 There results $m>0$.

Proof If $\lambda \neq 0$, and $u \in \mathcal{N}$, then

$$
\|u\|^{p}=|u|_{q}^{q}+\lambda|u|_{r}^{r} \leq C\left(\|u\|^{q}+\|u\|^{r}\right)
$$

namely $1 \leq C\left(\|u\|^{q-p}+\|u\|^{r-p}\right)$.

If $\|u\| \leq 1$ this implies $1 \leq 2 C\|u\|^{r-p}$. So that for all $u \in \mathcal{N},\|u\| \geq \min \left\{1,(2 C)^{-\frac{1}{r-p}}\right\}$. Therefore

$$
I(u)>\left(\frac{1}{p}-\frac{1}{r}\right)\|u\|^{p} \geq\left(\frac{1}{p}-\frac{1}{r}\right) \min \left\{1,(2 C)^{-\frac{1}{r-p}}\right\} .
$$

Lemma 3.4 There exists $u \in \mathcal{N}$ such that $I(u)=m$.

Proof First show that a minimizing sequence for $m$ in $\mathcal{N} \cap W_{r}$. To this aim, let $\left\{v_{k}\right\}_{k} \subseteq \mathcal{N}$ be a minimizing sequence. As usual we can assume $v_{k} \geq 0$. Let $w_{k}=v_{k}^{*} \in W_{r}$ be the non negative radial function given by Lemma 2.1. We have 


$$
\begin{gathered}
\left\|w_{k}\right\|^{p}=\int_{R^{N}}\left|\nabla v_{k}^{*}\right|^{p} d x \leq \int_{R^{N}}\left|\nabla v_{k}\right|^{p} d x=\int_{R^{N}}\left|v_{k}\right|^{q} d x+\lambda \int_{R^{N}}\left|v_{k}\right|^{r} d x \\
=\int_{R^{N}}\left|v_{k}^{*}\right|^{q} d x+\lambda \int_{R^{N}}\left|v_{k}^{*}\right|^{r} d x=\int_{R^{N}}\left|w_{k}\right|^{q} d x+\lambda \int_{R^{N}}\left|w_{k}\right|^{r} d x
\end{gathered}
$$

Hence if we set

$$
\gamma(t)=I^{\prime}\left(t w_{k}\right) t w_{k}=t^{q}\left\|w_{k}\right\|^{p}-t^{q}\left|w_{k}\right|_{q}^{q}-\lambda t^{r}\left|w_{k}\right|_{r}^{r}
$$

We have $\gamma(1) \leq 0$, while $\gamma(t) \geq 0$ for $t$ positive and small. Therefore there exist $t_{k} \in(0,1]$ such that $\gamma\left(t_{k}\right)=0$, that is

$t_{k} w_{k} \in \mathcal{N}$. We obtain

$$
\begin{gathered}
m \leq I\left(t_{k} w_{k}\right)=t_{k}^{p}\left(\frac{1}{p}-\frac{1}{r}\right)\left\|w_{k}\right\|^{p}+t_{k}^{q}\left(\frac{1}{r}-\frac{1}{q}\right)\left|w_{k}\right|_{q}^{q} \\
\leq\left(\frac{1}{p}-\frac{1}{r}\right)\left\|w_{k}\right\|^{p}+\left(\frac{1}{r}-\frac{1}{q}\right)\left|w_{k}\right|_{q}^{q} \\
\leq\left(\frac{1}{p}-\frac{1}{r}\right)\left\|v_{k}\right\|^{p}+\left(\frac{1}{r}-\frac{1}{q}\right)\left|v_{k}\right|_{q}^{q}=I\left(v_{k}\right)
\end{gathered}
$$

This implies that $\left\{t_{k} w_{k}\right\}_{k}$ is a minimizing sequence for $m$ and $t_{k} w_{k} \in W_{r}$, as we had claimed. In the sequel we set $u_{k}=t_{k} w_{k}$. Of course $u_{k} \geq 0$ and we can assume that up to subsequence, $u_{k} \rightarrow u$ in $W^{1, p}\left(R^{N}\right)$. By Lemma 2.3 we obtain $u_{k} \rightarrow u$ in $L^{q}\left(R^{N}\right)$ and in $L^{r}\left(R^{N}\right)$, and again up to subsequences $\quad, \quad u_{k} \rightarrow u$ almost everywhere, so that $u \geq 0$ a.e. and $u \in W_{r}$. Next prove that the weak limit $u$ belongs to $\mathcal{N}$ and $I(u)=m$. Let us first check that $u \in \mathcal{N}$. We have

$$
0 \leq C \leq\left\|u_{k}\right\|^{p}=\left|u_{k}\right|_{q}^{q}+\lambda\left|u_{k}\right|_{r}^{r}
$$

And passing to the limit, $0 \leq C \leq|u|_{q}^{q}+\lambda|u|_{r}^{r}$; this implies $u \neq 0$ and still from (7) $\|u\|^{p} \leq|u|_{q}^{q}+\lambda|u|_{r}^{r}$.If $\|u\|^{p}=|u|_{q}^{q}+\lambda|u|_{r}^{r}$, then $u \in \mathcal{N}$. So arguing by contradiction, assume that $\|u\|^{p}<|u|_{q}^{q}+\lambda|u|_{r}^{r}$. Defining as above, for $t>0$,

$$
\gamma(t)=I^{\prime}(t u) t u=t^{p}\|u\|^{p}-t^{q}|u|_{q}^{q}-\lambda t^{r}|u|_{r}^{r}
$$

From the above $\gamma(t)>0$ for small $t>0$ while $\gamma(1)<0$. So there is $t \in(0,1)$ such that $t u \in \mathcal{N}$. Hence,

$$
0<m \leq I(t u)=\left(\frac{1}{p}-\frac{1}{r}\right)\|t u\|^{p}+\left(\frac{1}{r}-\frac{1}{q}\right)|t u|_{q}^{q}
$$

$$
\begin{aligned}
& =t^{p}\left(\frac{1}{p}-\frac{1}{r}\right)\|u\|^{p}+t^{q}\left(\frac{1}{r}-\frac{1}{q}\right)|u|_{q}^{q}<\left(\frac{1}{p}-\frac{1}{r}\right)\|u\|^{p}+\left(\frac{1}{r}-\frac{1}{q}\right)|u|_{q}^{q} \\
& \leq \liminf _{k}\left(\frac{1}{p}-\frac{1}{r}\right)\left\|u_{k}\right\|^{p}+\lim _{k}\left(\frac{1}{r}-\frac{1}{q}\right)|u|_{q}^{q} \\
& =\liminf _{k}\left(\left(\frac{1}{p}-\frac{1}{r}\right)\left\|u_{k}\right\|^{p}+\left(\frac{1}{r}-\frac{1}{q}\right)|u|_{q}^{q}\right)=\liminf _{k} I\left(u_{k}\right)=m
\end{aligned}
$$

This contradiction proves that $\|u\|^{p}=|u|_{q}^{q}+\lambda|u|_{r}^{r}$, and therefore $u \in \mathcal{N}$. By the weak lower semicontinuity of the norm it is straightforward to deduce that $I(u) \leq \liminf _{k} I\left(u_{k}\right)=m$, and the lemma is proved.

Lemma 3.5 The minimum $u$ is a crucial point for $I$ in $W^{1, p}\left(R^{N}\right)$.

Proof Fix $v \in W^{1, p}\left(R^{N}\right)$ and $\varepsilon>0$ such that $u+s v \neq 0$ for all $s \in(-\varepsilon, \varepsilon)$. Define a function $\varphi:(-\varepsilon, \varepsilon) \times(0,+\infty) \rightarrow R$ by

$$
\begin{aligned}
& \varphi(s, t)=I^{\prime}(t(u+s v)) t(u+s v) \\
& =t^{p}\|u+s v\|^{p}-t^{q}|u+s v|_{q}^{q}-\lambda t^{r}|u+s v|_{r}^{r}
\end{aligned}
$$

Then $\varphi(0,1)=\|u\|^{p}-|u|_{q}^{q}-\lambda|u|_{r}^{r}=0$ and

$$
\frac{\partial \varphi}{\partial t}(0,1)=p\|u\|^{p}-q|u|_{q}^{q}-\lambda r|u|_{r}^{r}
$$

So, by the Implicit Function Theorem there exists a $\quad C^{1} \quad$ function $t:\left(-\varepsilon_{0}, \varepsilon_{0}\right) \rightarrow R \quad$ such that $t(0)=1$ and $\varphi(s, t(s))=0$ for all $s \in\left(-\varepsilon_{0}, \varepsilon_{0}\right)$. Defining $\gamma(s)=I(t(s)(u+s v))$, the function $\gamma$ is differentiable and has a minimum point at $s=0$; therefore

$$
0=\gamma^{\prime}(0)=I^{\prime}(t(0) u)\left(t^{\prime}(0) u+t(0) v\right)=I^{\prime}(u) v
$$

Since this holds for all $v \in W^{1, p}\left(R^{N}\right)$, then $I^{\prime}(u) v=0$.

\section{REFERENCES}

[1] J.P.Garcia Azorero, I.Peral Alonsl. Existence and nonuniqueness for the p-Lapacian: non-linear eigenvalues. Commun[J]. Partial Differ. Equ., 12(12),1389-1430,1987.

[2] P.Lindqvist. Notes on the p-Lapacian equation[R]. University of Fyväskylä , Department of Mathematics and Statistics, 102,2006.

[3] Fan Xian-ling, $\mathrm{Li}$ Zhan-cun. Linking and existence results for perturbations of the p-Laplacian[J]. Nonli. Anal., 42,1413-1420,2000.

[4] Liu Xia, Zhang Yuan-biao, Shi Hai-ping. Nonexistence and existence results for a fourth-order p-Laplacian discrete mixed boundary value problem[J]. Mediterranean Jour. Math., 12, 419-432,2015.

[5] Wang Xiao-zhi. A positive solution for some critical p-Laplacian systems[J]. Acta Math. Sin., 31, 479-500,2015. 
[6] Claudianor O.Alves, Giovany M. Figueiredo. Multiple solutions for a quasilinear Schrödinger equation on $R^{n}$ [J]. Acta Appl. Math., 136, 91-117,2015.

[7] H.Brzis, L.Nirenberg. Positive solutions of nonlinear elliptic equations involving critical Sobolev exponents[J]. Comm. Pure Appl. Math., 36, 437-477,1983.

[8] H.Brzis, L.Nirenberg, Remarks on finding critical points. Comm.Pure Appl. Math., 64, 939-963(1991).

[9] Cao Dao-min, Peng Shuang-jie. A note on the sign-changing solutions to elliptic problems with critical Sobolev and Hardy terms[J]. Journal Differ. Equ.,193, 424-434,2003.

[10] M.Willem. Minimax Theorems[M]. Progress in Nonlinear Differential Equations and Their Applications. vol. 24, Birkhäuser, Boston,1996.

[11] I.Kuzin, S.Pohozaev. Entire Solutions of Semilinear Elliptic Equations [M]. Progress in Nonlinear Diffnerential Equation and Their Applications, vol. 33 , Birkhäuser ,Basel, 1997.

[12] M.Struwe, A global compactness result for elliptic boundary value problems involving limiting nonlinearities[J]. Math. Z. 187(4), 511-517, 1984.

[13] M. Badiale, E.Serra. Semilinear Elliptic Equations for Beginners [M].Springer, London, 2011.

[14] H.Brezis, Analyse fonctionnelle[M]. Théorie et applications, Collection Mathématiques Appliquées pour la Maîtrise,Masson, Paris, 1983.

[15] E.H.Lieb, M.Loss, Analysis[M]. Graduate Studies in Mathematics, vol. 14,American Mathematical Society, Providence, 2001.

[16] Lawrence, C.Evans. Partial Differential Equations[M].Graduate Studies in Mathematics,vol.19,American of Mathmatical Society, Providence, 2010. 